\title{
Repurposed Drugs and Nutraceutical Supplements as Neglected Complements to Cancer Treatment
}

\author{
Tomas Koltai* \\ Independent researcher, Italy
}

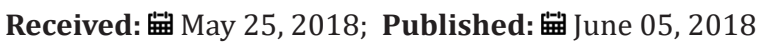

*Corresponding author: Tomas Koltai, Independent researcher, Florence, Italy

\begin{abstract}
In ovarian cancer there is formation of tumor cells in ovarian tissues. Lysophosphatidic acid (LPA) motivated cell proliferation, migration and endurance by acting on its cognate G-protein-coupled receptors. Lysophosphatidic acid (LPA), present in ascitic fluid, motivates the enlargement of malignant ovarian tumors by raising the appearance of vascular endothelial growth factor (VEGF) in ovarian cancer cells. Ovarian cancer cell progress is repressed by alendronate, a nitrogen containing biophosphate which attenuate the establishment of Rho by blocking the mevalonate pathway.
\end{abstract}

\section{Short Communication}

Cancer is still one of the major problems found in medical practice and in spite of clear advances in diagnosis and treatment, it remains an important cause of suffering and death in human and veterinary pathology. So there is a need to search for new approaches and ideas in this field. As of January 1, 2014, there were 14.5 million estimated survivors of the 10 most prevalent cancer sites among males and females in the United States [1]. One in four Americans will die of cancer [2] in the next years. Prevention, early diagnosis, surgery, radiotherapy, chemotherapy, immunotherapy and targeted drugs have made substantial contributions, and will continue to do so in the foreseeable future. But there is more that can be done. Since cancer is a very complex disease, it is reasonable to think that future solutions can only emerge from very sophisticated and expensive research. However, we dare to suggest that, right now, there are simple approaches that may be quite useful as complementary treatments in the management of cancer, just by re-positioning or repurposing, already approved existing drugs. This is the central idea (re-purposing existing drugs and associating them in a rational way), not as a core treatment of cancer, but as a complementary one, that we will consider in this article. As the burden of cancer keeps escalating worldwide, it is of utmost importance that efforts not be limited to finding new anticancer molecules at very high costs; they should also go into researching existing molecules which are used for other purposes and have shown anticancer activity. In certain cases of advanced disease, after the failure of intensive conventional therapy, complementary methods are the only remaining path.

Many cancer patients have initial success with surgery and chemo radiotherapy, successes which may last for variable lengths of time, only to find later that the cancer has become resistant to treatment. So adding inexpensive and well tolerated generic drugs, repurposed for the complementary treatment of cancer may, in certain cases, reverse treatment resistance. These complementary treatments may improve survival in cancer patients and even permit a reduction in chemo radiotherapy doses. Re-purposed drugs are drugs used in the treatment of diseases other than cancer, but have shown interesting anti-cancer effects. The advantages of re-purposed (or repositioned) drugs lay in the facts that they are fully known, their adverse effects have been thoroughly investigated, and they do not need expensive development and testing, so that they can quickly be added to anti-cancer treatment protocols. When repositioning an already-approved drug, there is no need to conduct phase I and phase IIa clinical trials. This is more likely to be the case for drugs being repurposed at similar or lower dosage than the one that has already been approved by regulatory agencies [3] For example, Astemizole is a potent secondgeneration antihistamine (histamine $\mathrm{H}-1$ receptor antagonist) that entered into medical practice in 1983. It was withdrawn from the US market in 1999 due to possible cardiac toxicity. Subsequently, it was identified as an inducer of cellular autophagy and cancer 
cell death in prostate cancer, acting synergistically with radiation. Enantiomers of NSAIDs naproxen and ketorolac block Rac and Cdc42 GTPase activation in response to growth factor stimulus and downstream cellular responses blocking cell proliferation, growth, and migration in xenograft models. These two NSAIDs are currently under investigation for use in ovarian cancer. In certain cases we have adequate knowledge about the anticancer activity of a drug, but there are still unresolved difficulties with pharmacokinetics. This is the case of curcumin which is not absorbed by the intestine due to its very low solubility. Success stories in drug repurposing for cancer are well known: for example thalidomide has been successfully re-purposed for leprosy and multiple myeloma; vitamin A derivatives (all trans retinoic acid) for promyelocytic leukemia [4]; everolimus for renal cancer; antimalarials like chloroquine and hydroxychloroquine against autophagy, etc.

The identification of possible anticancer candidates through massive screening methods like quantitative high-throughput screening or systematic repurposing screening in xenograft models [2] or computational methods are useful tools, but these methods do not avoid certain surprises in the in vivo setting when researchers find that the identified compound only works as an anticancer agent at toxic doses or is antagonistic to other chemotherapy drugs. This means that identification is only a necessary first step that must be followed by thorough and well designed experimental tests. When we analyze the medical literature we can find a lot of research concerning repurposed drugs for cancer, but little has come into practice. So we hope that this article may encourage more phase II medical trials and swiftly bring these drugs into everyday practice. Nowadays, drug development is mainly going into targeted therapies, but so far the results obtained have been modest or disappointing on overall survival in patients with solid tumors; side effects have been important and costs are excessively high [5]. Of course, there are exceptions, and a few targeted treatments have fully modified the outlook in certain tumors. This is the case of sorafenib and sutnitinib in renal cancer or imatinib in chronic myeloid leukemia. Cancer patients deserve that we use all the tools at our disposal, from classical rational drug design to observations in the laboratory, in the hospital, at the bedside and by the patients themselves. Drug repurposing or repositioning consists in the use of known drugs to treat a different disease from the one for which they were originally designed [6]. A very interesting example of a drug which is in the process of being repurposed is cariporide. This drug is an inhibitor of NHE-1, the sodium-hydrogen exchanger channel, that was held responsible for myocardial necrosis during ischemia-reperfusion episodes [7]. After many years of clinical trials it was concluded that cariporide did not provide the expected results. The Guardian trial, with more than 11,000 patients with unstable angina, failed to demonstrate any clinical benefit [8]. One very important finding that was overlooked in the Guardian trial report was the safety of the drug which was mentioned in the conclusions. Therefore, here we have a powerful inhibitor of
NHE-1 channels, whose dosage and lack of toxicity have been fully established, but it does not diminish the death toll of myocardial infarction. NHE-1 channels play a very significant role in cancer by creating intracellular alkaline and extracellular acidic environments that are necessary for malignant development. Harguindey et al. [9-10] tested cariporide for the treatment of cancer and they achieved very encouraging results. Cariporide has not entered clinical practice yet, but we are confident this will happen soon. Another interesting example is the case of artemisinin derivatives. The plant that is the source of these drugs, was used almost 2,000 years ago for the treatment of "fever" in China. In the 1970s it was re-discovered as a potent anti-malarial drug and has entered the clinical armamentarium as the number one medicine against malarial infection in almost the entire world (with the exception of the United States) [11]. Furthermore, artemisinin derivatives show strong anti-cancer effects, which are currently being actively researched. Sildenafil, the first drug discovered for the treatment of male erectile dysfunction, has found a place in the treatment of pulmonary hypertension. But, it seems that the history of sildenafil does not finish there: it also exhibits anti-cancer effects.

Many other compounds, like topiramate, acetazolamide, tolfenamic acid, metformin, orlistat, celecoxib, doxycycline, tolfenamic acid, sylimarin, etc. are waiting to be "re-discovered" as useful anti-cancer tools.

Sylimarin (sylibin) used for the treatment of toxic hepatic disease, like the one produced by Amanita phalloides intoxication, is a powerful down regulator of Stat3 [12]; NF-kB [13,14], Erb1 [15], induces down regulation of cyclin-dependent kinases and has shown in vitro and in vivo anti-cancer effects in renal carcinoma [16-18] and many other tumors [19-21]. Even though this seems to be the description of a powerful anti-cancer agent, devoid of toxicity, it has never been seriously tested on clinical grounds. There are a few compounds that are extensively used as "nutritional supplements", but in certain cases these compounds have anticancer abilities that have not been fully exploited. This is the case of evodiamine, dichloroacetate, genistein, quercetine, betulinic acid, lipoic acid, sulphoraphane, vinpocetine, epigallocatechin, and others. All these compounds deserve a little more attention. We also have to mention nonconventional ways of delivering treatments, specifically metronomic chemotherapy as opposed to the standard maximum chemotherapeutic dose schemes. The objective of this article is to call attention to the many other instruments that oncologist could use besides the approved and fairly well known standard treatments. We also encourage research and eventual clinical trials with these repurposed drugs and nutraceuticals because we are convinced that, at the end of the day, many of them will become useful instruments. Almost all the approaches and drugs mentioned here are compatible with the usual way of dealing with cancer, that is the reason why we call this presentation "complementary treatments": they are not supposed to replace any of the traditional approaches, but to increase their benefits. 


\section{References}

1. (2014) Cancer Prevalence: How Many People Have Cancer? American Cancer Society.

2. Roix JJ, Harrison SD, Rainbolt EA, Meshaw KR, Mc Murry AS, et al. (2014) Systematic Repurposing Screening in Xenograft Models Identifies Approved Drugs with Novel Anti-Cancer Activity. Plos One 9(8): e101708.

3. Oprea T, Bauman JE, Bologa CG, Buranda T, Chigaev A, et al. (2011) Drug repurposing from an academic perspective. Drug Discov Today Ther Strateg 8(3-4): 61-69.

4. Sanz MA (2006) Treatment of acute promyelocytic leukaemia. Blood 1: 147-155.

5. Pantziarka P, Bouche G, Meheus L, Sukhatme V, Sukhatme VP, et al. (2014) The repurposing drugs in oncology (ReDo) project. Ecancermedicalscience 8: 422.

6. Sleigh SH, Barton CL (2010) Repurposing Strategies for Therapeutics. Pharmaceutical Medicine 24(3): 151-159.

7. Karmazyn M (1999) The Role of the Myocardial Sodium-Hydrogen Exchanger in Mediating Ischemic and Reperfusion Injury: From Amiloride to Cariporide. Annals of the New York Academy of Sciences. 874(1): 326-334.

8. Theroux P, Chaitman BR, Danchin N, Erhardt L, Meinertz T, et al. (2000) Inhibition of the sodium-hydrogen exchanger with cariporide to prevent myocardial infarction in high-risk ischemic situations: main results of the Guardian trial. Circulation 102(25): 3032-3038.

9. Harguindey S, Arranz JL, Orozco JD, Rauch C, Fais S, et al. (2013) Cariporide and other new and powerful NHE1 inhibitors as potentially selective anticancer drugs-an integral molecular/biochemical/ metabolic/clinical approach after one hundred years of cancer research. Journal of translational medicine 11(1): 282.

10. Harguindey S, Orozco JP, Cuenca M, Fernández MC, Arranz JL (2014) New and powerful NHE1 inhibitors as potential anticancer drugs in bedside oncology: a prospective program of preclinical studies in cats and dogs with spontaneous malignant tumors. In Front Pharmacol. Conference Abstract: $4^{\text {th }}$ Annual Meeting of the International Society of Proton Dynamics in Cancer. Society of Proton Dynamics in Cancer.

11. (2015) WHO guidelines for the treatment of malaria. ( $3^{\text {rd }}$ edn), World Health Organization, Geneva, Switzerland.
12. Verdura S, Cuyàs E, Llorach Parés L, Pérez Sánchez A, Micol V, et al (2018) Silibinin is a direct inhibitor of STAT3. Food Chem Toxicol 116: 161-172.

13. Saliou C, Rihn B, Cillard J, Okamaoto T, Packer L (1998) Selective inhibition of NF-kB activation by the flavonoid hepatoprotector silymarin in HepG2. Evidence for different activating pathways. FEBS Letters 440(1-2): 8-12.

14. Manna SK, Mukhopadhyay A, Van NT, Aggarwal BB (1999) Silymarin suppresses TNF-induced activation of NF-kappa B, c-Jun N-terminal kinase, and apoptosis. J Immunol 163(12): 6800-6809.

15. Zi X, Grasso AW, Kung HJ, Agarwal R (1998) A flavonoid antioxidant, silymarin, inhibits activation of erbB1 signaling and induces cyclindependent kinase inhibitors, G1 arrest, and anticarcinogenic effects in human prostate carcinoma DU145 cells. Cancer Res 58(9): 1920-1929.

16. Liang L, Li L, Zeng J, Gao Y, Chen YL, et al. (2012) Inhibitory effect of silibinin on EGFR signal-induced renal cell carcinoma progression via suppression of the EGFR/MMP-9 signalling pathway. Oncol Rep 28(3): 999-1005.

17. Li L, Gao Y, Zhang L, Zeng J, He D, et al. (2008) Silibinin inhibits cell growth and induces apoptosis by caspase activation, down regulating surviving and blocking EGFR-ERK activation in renal cell carcinoma. Cancer Lett 272(1): 61-69.

18. Chang HR, Chen PN, Yang SF, Sun YS, Wu SW, et al. (2011) Silibinin inhibits the invasion and migration of renal carcinoma 786-0 cells in vitro, inhibits the growth of xenografts in vivo and enhances chemosensitivity to 5-fluorouracil and paclitaxel. Mol Carcinog 50(10): 811-823.

19. Sharma Y, Agarwal C, Singh AK, Agarwal R (2001) Inhibitory effect of silibinin on ligand binding to erbB1 and associated mitogenic signaling, growth, and DNA synthesis in advanced human prostate carcinoma cells. Mol Carcinog 30(4): 224-236.

20. Zhu W, Zhang JS, Young CY (2001) Silymarin inhibits function of the androgen receptor by reducing nuclear localization of the receptor in the human prostate cancer cell line LNCaP. Carcinogenesis 22(9): 13991403.

21. Zi X, Feyes DK, Agarwal R (1998) Anticarcinogenic effect of a flavonoid antioxidant, silymarin, in human breast cancer cells MDA-MB 468: induction of G1 arrest through an increase in Cip1/p21 concomitant with a decrease in kinase activity of cyclin-dependent kinases and associated cyclins. Clin Cancer Res 4(4): 1055-1064.

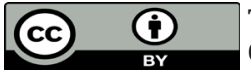

This work is licensed under Creative Commons Attribution 4.0 License

To Submit Your Article Click Here:

Submit Article

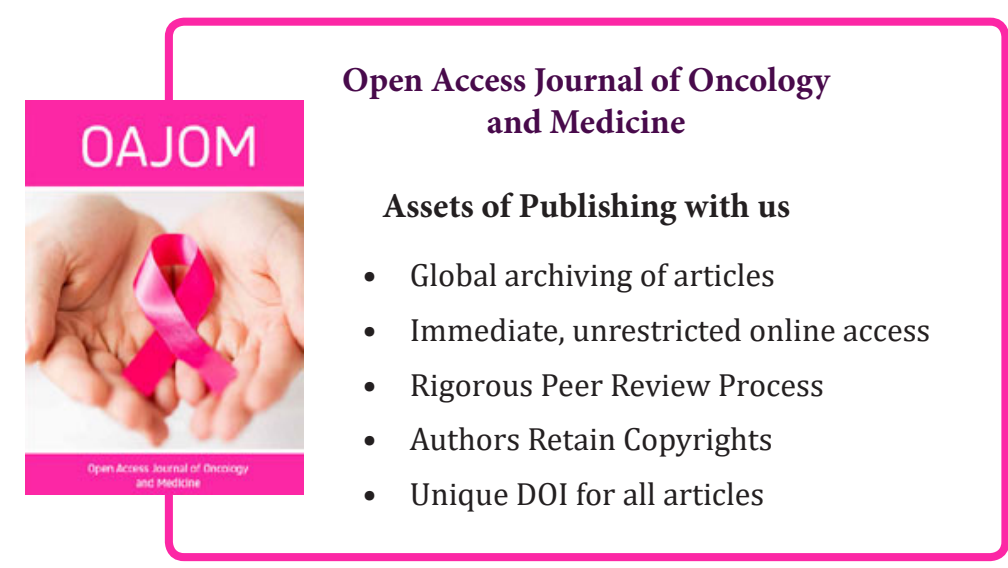

